\title{
THREE-DIMENSIONAL RAY TRACING AND GEOPHYSICAL INVERSION IN LAYERED MEDIA*
}

\author{
JOHN FAWCETT $\dagger$ AND H. B. KELLER $\dagger$
}

\begin{abstract}
In this paper the problem of finding seismic rays in a three-dimensional layered medium is examined. The "layers" are separated by arbitrary smooth interfaces that can vary in three dimensions. The endpoints of each ray and the sequence of interfaces it encounters are specified. The problem is formulated as a nonlinear system of equations and efficient, accurate methods of solution are discussed. An important application of ray tracing methods, which is discussed, is the nonlinear least squares estimation of medium parameters from observed travel times. In addition the "type" of each ray is also determined by the least squares process-this is in effect a deconvolution procedure similar to that desired in seismic exploration. It enables more of the measured data to be used without filtering out the multiple reflections that are not pure $\boldsymbol{P}$-waves.
\end{abstract}

Introduction. The seismic ray tracing problem has often been posed as a "shooting" problem (see for example Shah [6]). That is, the shooting angles of the ray at the source point are varied until the ray ends sufficiently close to the receiver. To find the seismic signals at an array of receivers, more sophisticated shooting methods have been devised (see for example Gjoystodtl and Ursin [2]). Our approach is to formulate the problem as a coupled system of nonlinear equations (Snell's law applied at each interface) and to solve this system by Newton's method. This is an extension to three dimensions of the work of Keller and Perozzi [3].

The travel times and ray amplitudes of the rays caused by a disturbance can be considered as functions of the parameters of the medium. Alternatively, using the ideas of nonlinear least squares estimation, we can estimate the parameters of the medium from observed ray travel times and amplitudes. This is an iterative algorithm and at each stage the rays for the current parameter estimates are determined. Least squares estimation of medium parameters has been discussed by various authors. Our threedimensional least squares methods are basically extensions of the work of Keller and Perozzi [3], [5]. In addition, however, we also determine the "type" of the rays as part of the inversion process. This is a completely new departure-a new type of deconvolution procedure-and it should be of great interest in exploration geophysics. It can enable the use of much significant data that is currently filtered out and thus discarded.

Formulation of the direct problem. We consider a layered medium, an example of which is shown in Fig. 1. The $i$ th interface is represented by a formula of the form:

$$
z=z_{i}(x, y) \equiv c_{i}+f_{i}(x, y) .
$$

Between consecutive interfaces is a homogeneous elastic medium with constant elastic wave speeds $v_{p}$ and $v_{s}$. The speeds may differ from one layer to the next. Since a ray segment in every layer is a straight line segment, any ray is determined by specifying its initial (source) point, $\mathbf{x}_{s}$, its final point $\mathbf{x}_{R}$, and in order, the $N$ points (nodes) at which it intersects the interfaces, say $\mathbf{x}_{1}, \mathbf{x}_{2}, \cdots, \mathbf{x}_{N}$. We adopt the notation that the $k$ th node, $\mathbf{x}_{k}$, lies on the interface $z_{i_{k}}, k=1,2, \cdots, N$. For each ray the integers $i_{1}$, $i_{2}, \cdots, i_{N}$ must be specified. The source and/or receiver points will often lie on the

\footnotetext{
* Received by the editors April 9, 1984. This research was supported by the U.S. Department of Energy Office of Basic Energy Sciences under contract DE-AS03-76SF 00767.

$\dagger$ Applied Mathematics, California Institute of Technology, Pasadena, California 91125.
} 


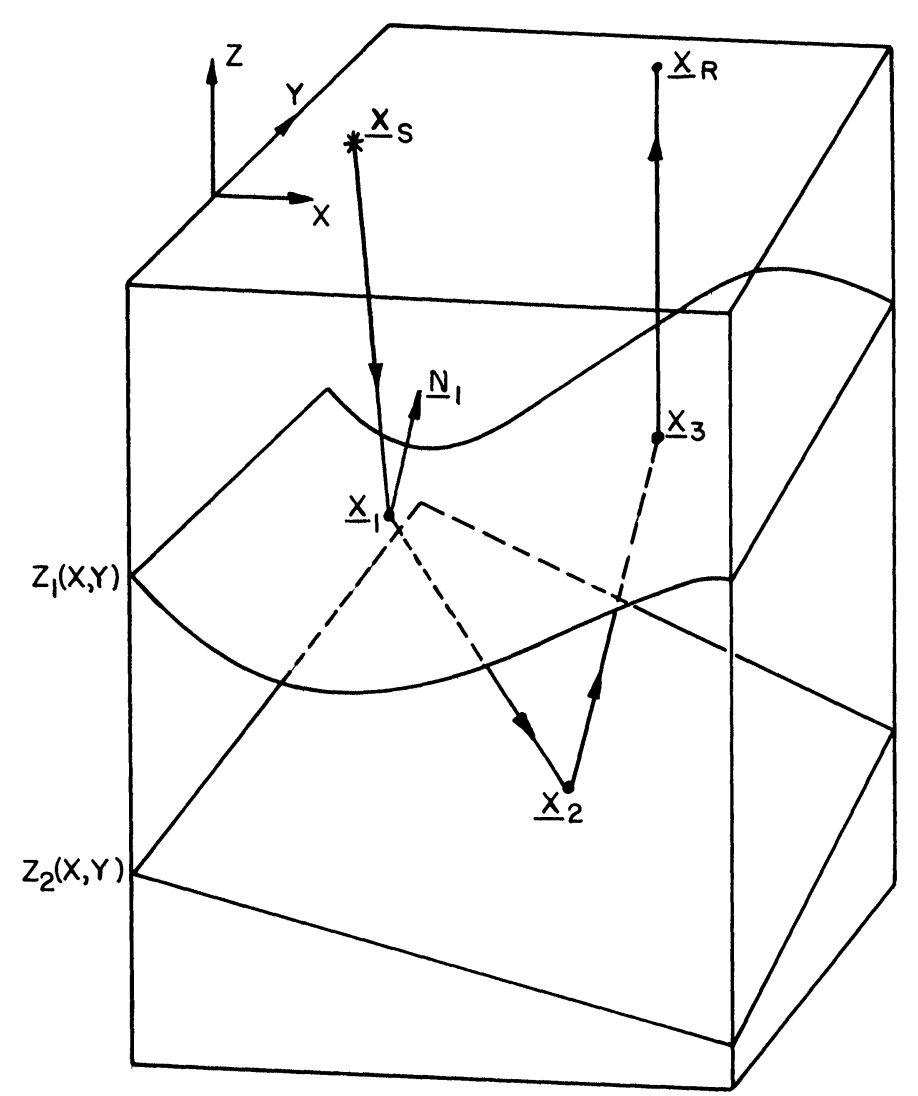

FIG. 1. Illustration of notation used.

earth's surface which is denoted as the interface $z_{0}$. A normal to the interface at the $k$ th node is:

$$
\mathbf{N}_{k} \equiv\left(\frac{-\partial z_{i_{k}}}{\partial x}, \frac{-\partial z_{i_{k}}}{\partial y}, 1\right)
$$

The vector $\mathbf{x}_{1}-\mathbf{x}_{s}$ denotes the ray segment from the source to the first node, and similarly for the other segments. We also use the notation:

$$
\begin{aligned}
& \mathbf{x}_{k} \equiv\left(x_{k}, y_{k}, z_{i_{k}}\left(x_{k}, y_{k}\right)\right), \quad k=1, \cdots, N, \\
& \mathbf{x}_{0} \equiv \mathbf{x}_{s}, \quad \mathbf{x}_{N+1} \equiv \mathbf{x}_{R}, \\
& d_{k} \equiv \sqrt{\left(\Delta x_{k}\right)^{2}+\left(\Delta y_{k}\right)^{2}+\left(\Delta z_{k}\right)^{2}}, \quad k=1, \cdots, N+1, \\
& \Delta x_{k} \equiv x_{k}-x_{k-1}, \quad \Delta y_{k} \equiv y_{k}-y_{k-1}, \quad \Delta z_{k} \equiv z_{i_{k}}-z_{i_{k-1}} .
\end{aligned}
$$

The velocity on the $k$ th ray segment is $v_{k}(k=1, \cdots, N+1)$ and it is also to be specified as either $v_{p}$ or $v_{s}$ (compressional or shear velocity) for the material comprising that layer.

At each node there are two unknowns, $x_{k}$ and $y_{k}$, with the " $z$ " coordinate implicitly known from $z=z_{i_{k}}\left(x_{k}, y_{k}\right)$ in (1). We write for the travel time, tr, along a ray, between a source, $\mathbf{x}_{s}$, and receiver $\mathbf{x}_{R}$ :

$$
\operatorname{tr}\left(\boldsymbol{x}_{s}, \mathbf{x}_{R} ; \mathbf{p}\right)=\sum_{k=1}^{N+1} \frac{d_{k}}{v_{k}}
$$


From Fermat's principle the travel time along a ray is extremal with respect to coordinate perturbations, and varying the $j$ th node, we obtain

$$
\begin{aligned}
& v_{j+1} v_{j} \frac{\partial \operatorname{tr}}{\partial x_{j}}=\frac{v_{j+1}}{d_{j}}\left(\Delta x_{j}+\frac{\partial z_{i_{j}}}{\partial x_{j}} \Delta z_{j}\right)-\frac{v_{j}}{d_{j+1}}\left(\Delta x_{j+1}+\frac{\partial z_{i_{j}}}{\partial x_{j}} \Delta z_{j+1}\right)=0, \\
& v_{j+1} v_{j} \frac{\partial \operatorname{tr}}{\partial y_{j}}=\frac{v_{j+1}}{d_{j}}\left(\Delta y_{j}+\frac{\partial z_{i_{j}}}{\partial y_{j}} \Delta z_{j}\right)-\frac{v_{j}}{d_{j+1}}\left(\Delta y_{j+1}+\frac{\partial z_{i_{j}}}{\partial y_{j}} \Delta z_{j+1}\right)=0 .
\end{aligned}
$$

Equations (5) are equivalent to the vector Snell's law at the $j$ th node:

$$
\frac{v_{j+1}}{d_{j}}\left(\mathbf{x}_{j}-\mathbf{x}_{j-1}\right) \times \mathbf{N}_{j}=\frac{v_{j}}{d_{j+1}}\left(\mathbf{x}_{j+1}-\mathbf{x}_{j}\right) \times \mathbf{N}_{j} .
$$

For $N$ nodes we have a vector of dimension $2 N$ of the unknown node coordinates:

$$
\mathbf{X} \equiv\left(x_{1}, y_{1}, x_{2}, y_{2}, \cdots, x_{N}, y_{N}\right) \text {. }
$$

However there are two scalar equations, (5a) and (5b), at each of the $N$ nodes, and we write this system of $2 N$ equations for $\mathbf{X}$ as:

$$
\mathbf{F}(\mathbf{X} ; \mathbf{p})=\mathbf{0} \text {. }
$$

Here $\mathbf{p}$ denotes a vector of parameters of interest to the problem. For example p might signify the source and receiver positions, layer velocities, or the geometry of the interfaces. We note from (5a) and (5b) that the first two components $F_{1}$ and $F_{2}$ involve $\mathbf{x}_{s}$ explicitly and $F_{2 N-1}, F_{2 N}$ involve $\mathbf{x}_{R}$ explicitly.

Solution of the ray equations. One commonly used method to solve a system such as (8) is Newton's method. If $\mathbf{X}^{(n)}$ is "sufficiently" close to the root of $\mathbf{F}$, then an improved value $\mathbf{X}^{(n+1)}$ is given by:

$$
\begin{aligned}
& \mathbf{X}^{(n+1)}=\mathbf{X}^{(n)}+\delta \mathbf{X}^{(n)}, \\
& \delta \mathbf{X}^{(n)}=-\left(J^{(n)}\right)^{-1} \mathbf{F}\left(\mathbf{X}^{(n)} ; \mathbf{p}\right) .
\end{aligned}
$$

Here, $J$ is the $2 N \times 2 N$ Jacobian matrix of the system: $J \equiv \partial F / \partial \mathbf{X}$. When the iterates in Newton's method are "close" to a simple root, they converge quadratically. However, in many problems, it may be difficult to supply a good initial guess, $\mathbf{X}^{(0)}$. To circumvent this difficulty we use continuation procedures.

Generation of initial (compressional) ray. To start the calculation of the rays for a given sequence of interfaces, we first calculate the purely compressional ray (i.e., each ray segment has the layer's compressional velocity) between the specified source and receiver. To provide a good initial estimate for this ray, we use a continuation method. Each interface is written in the form (1) where the constant $c_{i}$ represents a mean depth of the interface. Instead of using (1) we replace each interface by a family of interfaces:

$$
z=z_{i}(x, y \cdot \lambda) \equiv \lambda f_{i}(x, y)+c_{i}, \quad 0 \leqq \lambda \leqq 1 .
$$

Note that for $\lambda=0$ each interface becomes plane. We will solve the ray problem for the plane parallel interfaces $(\lambda=0)$ and then "deform" the plane interfaces into the desired (curved) interface $(\lambda=1)$ by letting $\lambda$ vary from 0 to 1 . The planar problem can be reduced to finding the zero of a scalar function (see [1, Appendix]). The system (8), with (1) replaced by (10) can be written as:

$$
\mathbf{F}(\mathbf{X} ; \lambda, \mathbf{p})=\mathbf{0} \text {. }
$$


If a ray solution to (11) is known for some value of $\lambda$, call it $\mathbf{X}=\mathbf{X}(\lambda)$, then as an initial ray estimate for the solution of (11) at $\lambda+\Delta \lambda$, we could take:

$$
\mathbf{X}^{(0)}(\lambda+\Delta \lambda)=\mathbf{X}(\lambda) \text {. }
$$

However since le can calculate $\dot{\mathbf{X}}(\lambda) \equiv d \mathbf{X} / d \lambda$, we can obtain a better, second order accurate approximation:

$$
\mathbf{X}^{(0)}(\lambda+\Delta \lambda)=\mathbf{X}(\lambda)+\Delta \lambda \dot{\mathbf{X}}(\lambda) .
$$

In practice, to minimize the amount of computation, we use (12a) or (12b) with $\lambda=0$ and $\Delta \lambda=1$. In most of the numerical examples this value, $\Delta \lambda=1$, leads to a quadratically convergent sequence of iterates. If, however, Newton's method does not converge quickly (say 7 iterations), we return to our formulae with $\lambda_{1}=0, \lambda_{2}=\frac{1}{2}$, and $\lambda_{3}=1$ $\left(\Delta \lambda=\frac{1}{2}\right)$. In general, if necessary, one can decrease $\Delta \lambda$ to some user input minimum, in order that (12a) or (12b) eventually lead to a convergent sequence at $\lambda=1$.

Continuation in parameter values. As indicated previously we may wish to calculate the rays for different values of the parameter vector $\mathbf{p}$ appearing in (8). If $\mathbf{p}^{(0)}$ and $\mathbf{p}^{(1)}$ are two parameter vectors for which we wish to solve (8) we introduce:

$$
\mathbf{p}(\lambda)=(1-\lambda) \mathbf{p}^{(0)}+\lambda \mathbf{p}^{(1)}, \quad 0 \leqq \lambda \leqq 1 .
$$

With this family replacing $\mathbf{p}$ in (8) we now use continuation in $\lambda$ as discussed above.

The parameter dependence of the ray solution $\mathbf{X}$ is indicated by writing $\mathbf{X}=\mathbf{X}(\mathbf{p})$ and so (8) becomes:

$$
\mathbf{F}(\mathbf{X}(\mathbf{p}) ; \mathbf{p})=\mathbf{0} \text {. }
$$

This relation is an identity in $\mathbf{p}$. If $\mathbf{x}(\mathbf{p})$ depends smoothly upon $\mathbf{p}$, then:

$$
\frac{d \mathbf{F}}{d \mathbf{p}}=\frac{\partial \mathbf{F}}{\partial \mathbf{X}} \frac{\partial \mathbf{X}}{\partial \mathbf{p}}+\frac{\partial \mathbf{F}}{\partial \mathbf{p}}=\mathbf{0}
$$

or

$$
\frac{\partial \mathbf{X}}{\partial \mathbf{p}}=-\mathbf{J}^{-1} \frac{\partial \mathbf{F}}{\partial \mathbf{p}} .
$$

For continuation in a scalar parameter, $\lambda$, we get from (15) that:

$$
\dot{\mathbf{X}}(\lambda)=-\mathbf{J}^{-1} \frac{\partial \mathbf{F}}{\partial \lambda} \text {. }
$$

To get rays of different types (i.e., mixed $P$ and $S$ waves) we use continuation in the wave speeds employing the above technique. The details are given in Keller and Perozzi [3].

Inverse problems. We have discussed above methods for calculating a ray between a specified source and receiver. Once a ray has been found, the travel time can be calculated from (3). Also, the geometric optics amplitude can be calculated exactly, but we will not discuss these calculations here (see Fawcett [1]).

We now suppose that we have $M$ observed travel times for various rays between different source/receiver pairs which are denoted by the vector obs. Using this vector, we wish to estimate the $K$-dimensional parameter vector, p, appearing in (8) and (4). To estimate $\mathbf{p}$ we seek to minimize

$$
\min _{\mathbf{p}}\left\|\operatorname{tr}\left(\mathbf{x}_{s}, \mathbf{x}_{R} ; \mathbf{p}\right)-\mathbf{o b s}\right\|_{2}
$$


Here $\operatorname{tr} \equiv\left(\operatorname{tr}_{1}, \operatorname{tr}_{2}, \cdots, \operatorname{tr}_{M}\right)$ where $\operatorname{tr}_{i}$ is the calculated travel time using the parameter estimate, $\mathbf{p}$, corresponding to the $i$ th ray. The parameter vector $\mathbf{p}$ can be, for example, the encountered layer velocities or the dips of the encountered interfaces. Also, as is shown in Example 3 and in Fawcett [1], p can include unknown ray types (i.e., $P P P \cdots P, P S P \cdots P, \cdots$, etc. where a " $P$ " denotes a compressional ray segment and a " $S$ " a shear ray segment). We use the notation $\mathbf{t r}-\mathbf{o b s} \equiv \mathbf{R}(\mathbf{p})$ and seek a minimizing $\mathbf{p}_{0}$ for (17) by standard nonlinear least squares techniques. In particular the GaussNewton method yields the iteration scheme:

$$
\begin{aligned}
\mathbf{p}^{(n+1)} & =\mathbf{p}^{(n)}+\Delta \mathbf{p}^{(n)}, \\
\Delta \mathbf{p}^{(n)} & =-\left(J_{L}^{T} J_{L}\right)^{-1} J^{T} \mathbf{R}\left(\mathbf{p}^{(n)}\right)
\end{aligned}
$$

where $J_{L} \equiv\left(\partial R_{i} / \partial p_{j}\right)$ is an $M$ by $K$ matrix. It is often advantageous to damp the Newton step, and take

$$
\Delta \mathbf{p}^{(n)}=-\left(J_{L}^{T} J_{L}+\sigma_{n} I\right)^{-1} J_{L}^{T} \mathbf{T}\left(\mathbf{p}^{(n)}\right) .
$$

Here the choice of $\sigma_{n}$ is somewhat ad hoc, but basically $\sigma_{n}$ is chosen so that $\left\|\mathbf{R}\left(\mathbf{p}^{(n+1)}\right)\right\|_{2}<\left\|\mathbf{R}\left(\mathbf{p}^{(n)}\right)\right\|_{2}$. The iteration scheme in (18) is equivalent to solving a sequence of linear least squares problems. To solve each linear problem, we use the well-established theory of linear least squares (see for example Lawson and Hanson [4]). For each value of $\mathbf{p}^{(n)}$ in (18), we must find all $M$ rays of the system. Thus the efficiency and accuracy of the ray-tracing method is very important. This is one of the main reasons for the development of fast ray-tracing methods.

\section{Numerical examples.}

Example 1. Some ray calculations. In this example we consider two interfaces:

$$
\begin{aligned}
& z_{1}(x, y) \equiv \frac{x^{3}}{10}+\frac{y^{3}}{20}-\frac{x}{5}+\frac{y}{4}-5 \\
& z_{2}(x, y) \equiv-\frac{x^{2}}{20}-\frac{y^{2}}{10}-10 .
\end{aligned}
$$

The layer velocities are $v_{p, 1}=6, v_{s, 1}=3$ in $0<z<z_{1}(x, y)$ and $v_{p, 2}=8$, and $v_{s, 2}=4$ in $z_{1}(x, y)<z<z_{2}(x, y)$. We calculate the purely compressional ray from source $\mathbf{x}_{s} \equiv$ $(0,0,0)$ to $\mathbf{x}_{R} \equiv(1,1,0)$. The ray is shown schematically, in Fig. 2 . We show the various stages of calculation below in Tables $1 \mathrm{a}-1 \mathrm{c}$. Table 1 a shows the " $x$ " and " $y$ " coordinates

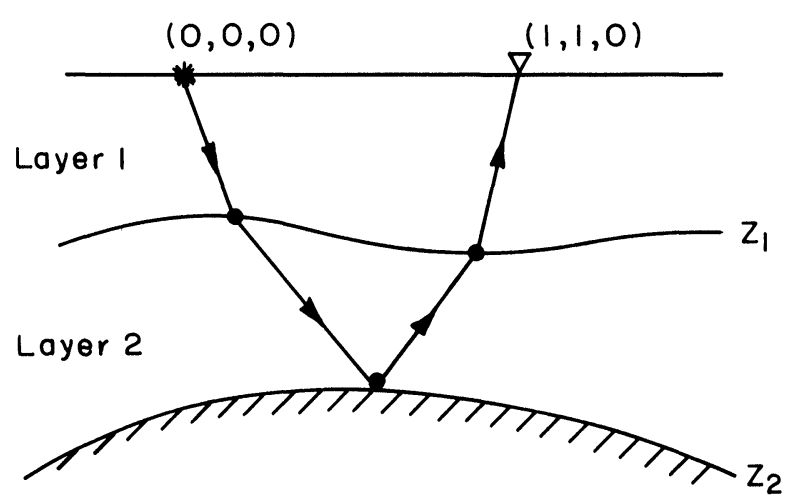

FIG. 2. Schematic cross-section of ray. 
TABLE la

Planar solution.

\begin{tabular}{ccc}
\hline node & " $x "$ & " $y "$ \\
\hline 0 & 0.000000 & 0.000000 \\
1 & 0.214111 & 0.214111 \\
2 & 0.500000 & 0.500000 \\
3 & 0.785889 & 0.785889 \\
4 & 1.000000 & 1.000000 \\
\hline
\end{tabular}

TABLE $1 \mathrm{~b}$

Initial ray estimate.

\begin{tabular}{crr}
\hline node & " $x "$ & \multicolumn{1}{c}{$y "$} \\
\hline 0 & 0.000000 & 0.000000 \\
1 & -0.163438 & 0.182553 \\
2 & -0.067111 & -0.001901 \\
3 & 0.539834 & 0.824263 \\
4 & 1.000000 & 1.000000 \\
\hline
\end{tabular}

TABLE 1c

Ray solution, velocities: $6,8,8,6$.

\begin{tabular}{crc}
\hline node & " $x "$ & " $y "$ \\
\hline 0 & 0.000000 & 0.000000 \\
1 & -1.069961 & 0.326969 \\
2 & 0.169430 & 0.329889 \\
3 & 0.594940 & 1.026166 \\
4 & 1.000000 & 1.000000 \\
\hline
\end{tabular}

TABLE 1d

Estimated ray 6, 4, 8, 6.

\begin{tabular}{ccr}
\hline node & " $x "$ & \multicolumn{1}{c}{ " $y "$} \\
\hline 0 & 0.000000 & 0.000000 \\
1 & 0.201051 & -0.037784 \\
2 & 0.209590 & 0.178123 \\
3 & 0.612635 & 0.947115 \\
4 & 1.000000 & 1.000000 \\
\hline
\end{tabular}

TABLE 1e

Ray solution, velocities 6, 4, 8, 6 .

\begin{tabular}{ccr}
\hline node & " $x "$ & \multicolumn{1}{c}{ " $y "$} \\
\hline 0 & 0.000000 & 0.000000 \\
1 & 0.346168 & -0.214290 \\
2 & 0.298160 & 0.069099 \\
3 & 0.661044 & 0.891464 \\
4 & 1.000000 & 1.000000 \\
\hline
\end{tabular}


of the ray solution, $\mathbf{x}(\lambda)$, for plane interfaces at $z=-5$ and $z=-10$ corresponding to $\lambda=0$. Table $1 \mathrm{~b}$ shows the initial ray estimate $\mathbf{x}(0)+d \mathbf{x}(0) / d \lambda$. With this initial estimate we have a residual $\|\mathbf{F}(\mathbf{x})\|_{2}=1.80$. The two successive Newton iterations had residuals: $1.26 \times 10^{-2}$ and $6.56 \times 10^{-6}$. The resulting solution is shown in Table 1c. Next we seek the ray in which the second segment propagates in shear. Thus we set $\mathbf{p}(\lambda)=$ $(1-\lambda) \mathbf{v}_{1}+\lambda \mathbf{v}_{2}$, where $\mathbf{v}_{1}$ is the purely compressional velocity sequence and $\mathbf{v}_{2} \equiv$ $\left[v_{p, 1}, v_{s, 2}, v_{p, 2}, v_{p, 1}\right]$. Using this $\mathbf{p}(\lambda)$ and the $L U$ decomposition of the Jacobian, $J$, from the next to last Newton iteration, we estimate the ray solution as, $\mathbf{x}^{(0)}=\mathbf{x}(0)+d \mathbf{x}(0) / d \lambda$. This estimate is shown below in Table 1d. It has a residual of $\|\mathbf{F}\|_{2}=.3633$. The next two Newton iterations had residuals of $1.65 \times 10^{-3}$ and $3.08 \times 10^{-5}$. The resulting ray solution is shown in Table 1e. We also calculated the rays to receivers at $\mathbf{x}_{R} \equiv(2,2,0)$ and $\mathbf{x}_{R} \equiv(3,3,0)$. The purely compressional rays are shown for the continuation parameter values $\lambda=0, \lambda=\frac{1}{2}$, and $\lambda=1$ in Figs. 3a-3c. The $\lambda=\frac{1}{2}$ problem is shown only for illustration since, as discussed above, $\Delta \lambda=1$ was sufficient.

Example 2. Inversion for interface shape and layer velocities. We start with the following interfaces:

$$
\begin{aligned}
& z_{1} \equiv .08 x_{1}+.1 y_{1}-5 \\
& z_{2} \equiv .2 x_{2}+.1 y_{2}-10
\end{aligned}
$$

The layer velocities are: $v_{p, 1}=12, v_{s, 1}=6, v_{p, 2}=14$, and $v_{s, 2}=8.2$. We generate the travel times for the rays of types $(P, P),(P, S),(P, P, P, P)$ and $(P, S, P, P)$ for a source at the origin and receivers placed at: $(x= \pm i, y=i, z=0), i=1,2,3,4,5$. One such set of rays for $i=1$ is shown schematically, in Fig. 4.

For the inverse problem we consider the layer velocities $\left(v_{p, 1}, v_{s, 1}, v_{p, 2}, v_{s, 2}\right)$ and the coefficients of the interfaces $z_{1}=\alpha_{1} x+\beta_{1} y+\gamma_{1}$ and $z=\alpha_{2} x+\beta_{2} y+\gamma_{2}$ are unknown. Below in Table 2 we show the results of the inversion. Numerically, we traced all the rays using our method described above. The least squares Jacobian, $\left(\partial R_{i} / \partial p_{j}\right)$, is formed analytically. Here

$$
\frac{\partial R_{i}}{\partial V}=-\sum_{j=1}^{N+1} \frac{D_{j}}{v_{j}^{2}} \delta_{j, k}
$$

where $\delta_{j, k}=1$ if $v_{j}$ corresponds to same layer and velocity type $(P$ or $S)$ as $V ; \delta_{j, k}=0$ otherwise. $V$ could be $v_{p, 1}, v_{s, 1}, v_{p, 2}$ or $v_{s, 2}$. Also

$$
\begin{aligned}
& \frac{\partial R_{i}}{\partial \alpha_{k}}=\sum_{j=1}^{N+1} \Delta z_{j} \frac{\left(\tau_{j, k} x_{j}-\Omega_{j, k} x_{j-1}\right)}{D_{j} v_{j}}, \\
& \frac{\partial R_{i}}{\partial \beta_{k}}=\sum_{j=1}^{N+1} \Delta z_{j} \frac{\left(\tau_{j, k} y_{j}-\Omega_{j, k} y_{j-1}\right)}{D_{j} v_{j}},
\end{aligned}
$$

and

$$
\frac{\partial R_{i}}{\partial \gamma_{k}}=\sum_{j=1}^{N+1} \frac{\Delta z_{j}\left(\tau_{j, k}-\Omega_{j, k}\right)}{D_{j} v_{j}} .
$$

Here $\tau_{j, k}$ equals 1 if $\mathbf{x}_{j}$ is on the $k$ th interface and zero otherwise and $\Omega_{j, k}=1$ if $\mathbf{x}_{j-1}$ is on the $k$ th interface and zero otherwise. All quantities, $D_{j}, \mathbf{x}_{j}$ etc., are evaluated for the $i$ th ray. Thus we see that even starting far away from the known parameter values $\mathbf{p}^{*}$ we converge in 5 iterations (we are close after only 2 or 3 iterations) to $\mathbf{p}^{*}$. 


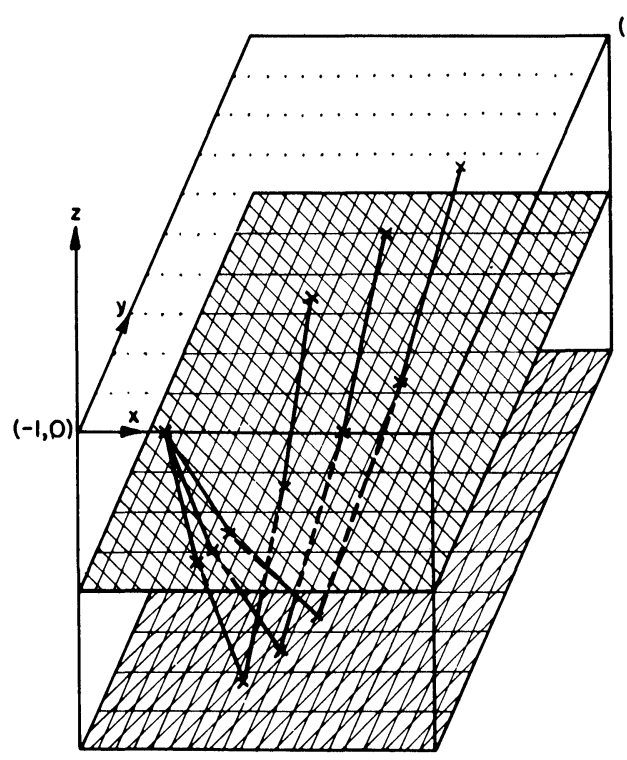

FIG. 3a. Rays for example $1 ; \lambda=0$.

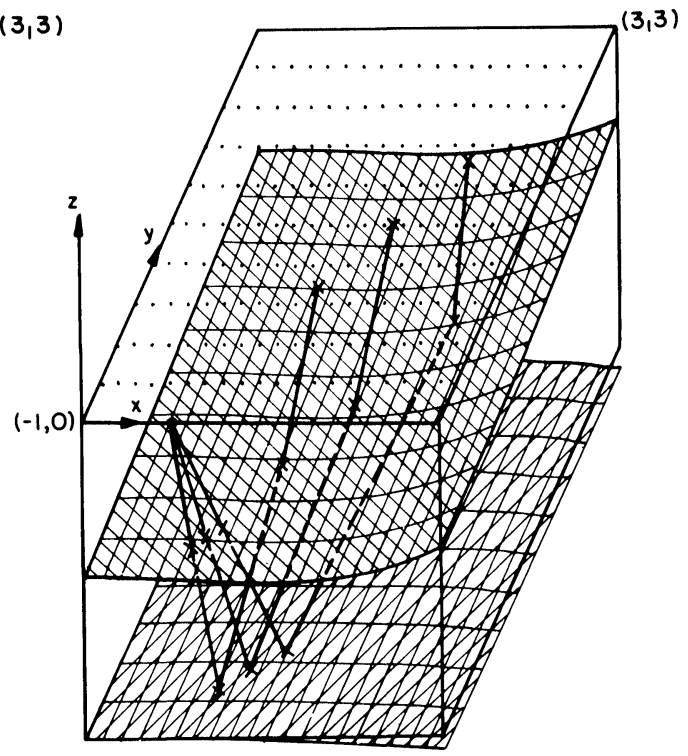

FIG. 3b. Rays for example $1 ; \lambda=\frac{1}{2}$.

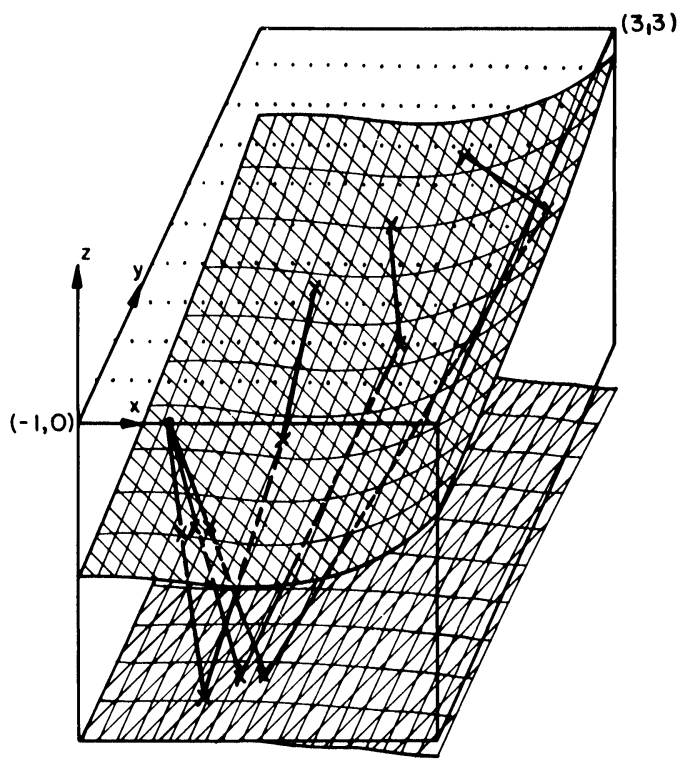

FIG. 3c. Rays for example $1 ; \lambda=1$. 
TABLE 2

Velocity/interface inversion: 2 arrays.

\begin{tabular}{cccccc}
\hline it \# & Velocities & \multicolumn{1}{c}{ Interfaces } & Residual & Gradient & $S_{\min }$ \\
\hline \multirow{2}{*}{0} & $(8.000,4.000)$ & $(.000,0.000,-4.00)$ & 1.28 & 3.781 & $3.6 \mathrm{E}-3$ \\
& $(16.00,7.000)$ & $(.000,0.000,-8.00)$ & & & \\
1 & $(10.56,5.283)$ & $(.053, .062,-4.59)$ & .287 & .598 & $2.3 \mathrm{E}-3$ \\
& $(15.99,9.526)$ & $(.196, .118,-10.08)$ & & & \\
2 & $(11.82,5.917)$ & $(.077,-.097,-4.94)$ & .034 & .065 & $2.7 \mathrm{E}-3$ \\
& $(14.09,8.259)$ & $(.197, .124,-10.03)$ & & & $2.6 \mathrm{E}-3$ \\
3 & $(12.00,5.999)$ & $(.080,-.100,-5.00)$ & $1.6 \mathrm{E}-3$ & $3.6 \mathrm{E}-3$ & \\
& $(14.00,8.203)$ & $(.200, .010,-10.00)$ & & & \\
& $(12.000,6.000)$ & $(.080,-.100,-5.00)$ & $4.6 \mathrm{E}-4$ & $9.0 \mathrm{E}-4$ & \\
& $(14.000,8.2000)$ & $(.200, .100,-10.00)$ & & & \\
\hline
\end{tabular}

Final estimates: velocities: (12.00001, 6.000006, 13.99997, 8.1999982);

interfaces: $(8.0000073 \mathrm{E}-2,-.100006,-5.000005)(.1999997,9.9999577 \mathrm{E}-2,-9.999994)$;

residual: $2.02 \mathrm{E}-6$; gradient: $2.18 \mathrm{E}-6$.

Example 3. Determination of ray types. We start with the following interfaces:

$$
\begin{aligned}
& z_{1} \equiv-\frac{x_{1}^{2}}{18}+\frac{y_{1}}{20}-5, \\
& z_{2} \equiv \frac{x_{2}}{16}-\frac{y_{2}}{10}-10 .
\end{aligned}
$$

The layer velocities are: $v_{p, 1}=5, v_{s, 1}=2.9, v_{p, 2}=7, v_{s, 2}=4$. We generate the travel times for the rays of type $(P, P),(P, S),(P, P, P, P),(P, S, P, P),(P, S, S, P)$, and $(P, P, S, P)$, for a source at the origin and receivers at $(x=y=i, z=0)$, for $i=1, \cdots, 5$. We shall now suppose that at receiver " $i$ " we have a set of observed travel times $\mathscr{T}=\left\{\operatorname{tr}_{1}, \operatorname{tr}_{2_{i}}, \operatorname{tr}_{3_{i}}\right\}$. But it is unknown which ray type of the set $\mathscr{R} \equiv\{(P, S, P, P),(P, S, S, P),(P, P, S, P)\}$ to associate with each of these travel times. Physically, as $v_{s, k}<v_{p, k}$ one probably could distinguish $(P, S, S, P)$ but for the sake of illustration we consider the above case. Thus, in this example, each travel time from $\mathscr{T}$ has one of three possible ray types. To estimate $v_{s, 2}$ we must include at least some of the observations from $\mathscr{T}$. A "brute force" method for including this information would be to try every possible labelling of the travel times of $\mathscr{T}$, to determine the best inversion result. This, however, would require $3^{3}=27$ inversions. A much more efficient method, for the inclusion of the information of $\mathscr{T}$, is based upon the following observations.

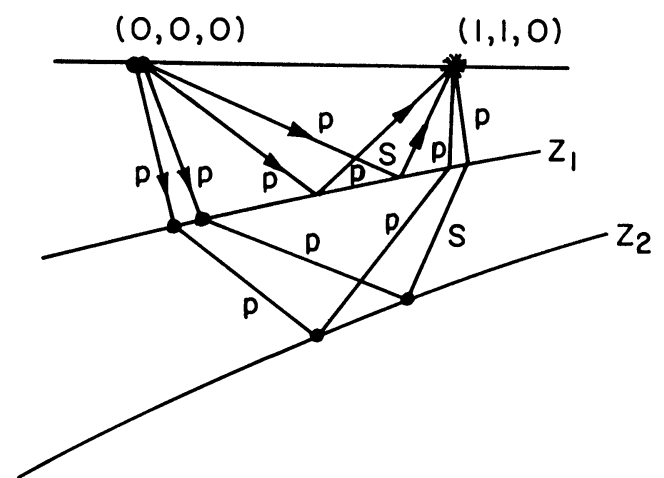

FIG 4. Schematic cross-section of rays used in inversion. 
In the inversion procedure, the travel times for the ray types of $\mathscr{R}$ are calculated, for each receiver $i$, for the current parameter estimate. After the calculation of the rays we wish to form the residual vector, $\mathbf{R}$, with components:

$$
\begin{aligned}
& R_{1_{i}} \equiv \operatorname{tr}_{1_{i}}^{c} \text {-observed travel time }(P, S, P, P), \\
& R_{2_{i}} \equiv \operatorname{tr}_{2_{i}}^{c} \text {-observed travel time }(P, S, S, P), \\
& R_{3_{i}} \equiv \operatorname{tr}_{3_{i}}^{c} \text {-observed travel time }(P, P, S, P) .
\end{aligned}
$$

Here $\operatorname{tr}_{1_{i}}^{c}$ is the calculated travel time for $(P, S, P, P)$ at receiver $i$, etc. However, we cannot form $\mathbf{R}$ as we do not know which arrangement of $\mathscr{T}$ to associate with the ray types of $\mathscr{R}$. To circumvent this problem, we rewrite (20) as:

$$
\begin{aligned}
& R_{1_{i}} \equiv \operatorname{tr}_{1_{i}}^{c}-\alpha_{1} \operatorname{tr}_{1_{i}}-\alpha_{2} \operatorname{tr}_{2_{i}}-\left(1-\alpha_{1}-\alpha_{2}\right) \operatorname{tr}_{3_{i}}, \\
& R_{2_{i}} \equiv \operatorname{tr}_{2_{i}}^{c}-\beta_{1} \operatorname{tr}_{1_{i}}-\beta_{2} \operatorname{tr}_{2_{i}}-\left(1-\beta_{1}-\beta_{2}\right) \operatorname{tr}_{3_{i}}, \\
& R_{3_{i}} \equiv \operatorname{tr}_{3_{i}}^{c}-\left(1-\alpha_{1}-\beta_{1}\right) \operatorname{tr}_{1_{i}}-\left(1-\alpha_{2}-\beta_{2}\right) \operatorname{tr}_{2_{i}}-\left(\alpha_{1}+\beta_{1}+\alpha_{2}+\beta_{2}-1\right) \operatorname{tr}_{3_{i}} .
\end{aligned}
$$

Thus, we have introduced 4 new unknown parameters into the inversion $\left(\alpha_{1}, \alpha_{2}, \beta_{1}, \beta_{2}\right)$. We assume that these parameters should be a sequence of ones and zeros and this is reflected in the form of the linear combinations in (21). In this example, we use the same coefficients at each station; thus we are assuming that by some means (perhaps by establishing some trends in the data) we have been able to arrange the observations for all stations into groups of unknown ray type. In general, however, we can introduce new coefficients for specific receivers, if we so desire.

The 4 new parameters result in 4 new columns in the least squares Jacobian, with the following nonzero elements:

$$
\begin{array}{ll}
\frac{\partial R_{1_{i}}}{\partial \alpha_{1}}=\operatorname{tr}_{3_{i}}-\operatorname{tr}_{1_{i}}, & \frac{\partial R_{1_{i}}}{\partial \alpha_{2}}=\operatorname{tr}_{3_{i}}-\operatorname{tr}_{2_{i}}, \\
\frac{\partial R_{3_{i}}}{\partial \alpha_{1}}=\operatorname{tr}_{1_{i}}-\operatorname{tr}_{3_{i}}, & \frac{\partial R_{3_{i}}}{\partial \alpha_{2}}=\operatorname{tr}_{2_{i}}-\operatorname{tr}_{3_{i}}, \\
\frac{\partial R_{2_{i}}}{\partial \beta_{1}}=\operatorname{tr}_{1_{i}}-\operatorname{tr}_{1_{i}}, & \frac{\partial R_{3_{i}}}{\partial \beta_{2}}=\operatorname{tr}_{3_{i}}-\operatorname{tr}_{2_{i}}, \\
\frac{\partial R_{3_{i}}}{\partial \beta_{1}}=\operatorname{tr}_{1_{i}}-\operatorname{tr}_{3_{i}}, & \frac{\partial R_{3_{i}}}{\partial \beta_{2}}=\operatorname{tr}_{2_{i}}-\operatorname{tr}_{3_{i}}
\end{array}
$$

In Table 3 below, we use the $(P, S),(P, P)$ and $(P, P, P, P)$ travel times at the 5 receivers, and also the travel times for $(P, S, P, P),(P, S, S, P),(P, P, S, P)$ with incorrect labels $\alpha_{1}=\alpha_{2}=\beta_{1}=\beta_{2}=.3$. Thus all the initial ray types are incorrectly identified. At the fifth iteration, the ray types are correctly identified, and the velocities correctly estimated.

If we add $1 \%$ random noise to the observed travel times, for this example, the noise level is greater than the difference in travel times for the $(P, S, P, P)$ and $(P, P, S, P)$ rays. The $(P, S, S, P)$ travel time is still distinguishable. If we redo the inversion with this data, with the same initial guess as above, we arrive at the estimate:

$$
\begin{aligned}
& V_{p, 1}=5.04, \quad V_{s, 1}=2.91, \quad V_{p, 2}=6.97, \quad V_{s, 2}=3.98, \\
& \alpha_{1}=.769, \quad \alpha_{2}=1.46 \times 10^{-2}, \quad \beta_{1}=.1464, \quad \beta_{2}=.996, \\
& \text { residual }=.147, \quad \text { gradient }=4.41 \times 10^{-6} .
\end{aligned}
$$


TABLE 3

Inversion for velocity and ray type.

\begin{tabular}{ccccc}
\hline it \# & Velocities & Labels & Residual & Gradient \\
\hline 0 & $(8.00,4.00)$ & $(.300, .300)$ & 10.38 & 15.46 \\
& $(12.00,6.00)$ & $(.300, .300)$ & & \\
1 & $(3.20,2.49)$ & $(.977,1.45 \mathrm{E}-4)$ & 16.08 & 124.19 \\
& $(3.43,3.00)$ & $(1.32 \mathrm{E}-2,1.000)$ & & \\
2 & $(4.35,2.85)$ & $(.989,1.86 \mathrm{E}-4)$ & 4.57 & 18.65 \\
& $(7.86,5.67)$ & $(-2.25 \mathrm{E}-2,1.000)$ & & \\
3 & $(4.92,2.90)$ & $(.995,9.74 \mathrm{E}-5)$ & .728 & 2.134 \\
& $(6.53,3.99)$ & $(-8.34 \mathrm{E}-3,1.000)$ & & \\
4 & $(5.00,2.90)$ & $(1.00,1.02 \mathrm{E}-5)$ & $3.73 \mathrm{E}-2$ & $9.36 \mathrm{E}-2$ \\
& $(6.97,4.00)$ & $(-1.28 \mathrm{E}-3,1.00)$ & & \\
\hline
\end{tabular}

Final estimate: velocities: $(5.000000,2.900000,6.999860,4.000000)$; labels: $(1.000008,2.164 \mathrm{E}-7,-1.095 \mathrm{E}-5, .999999)$; residual: $1.56 \mathrm{E}-4$; gradient: $3.78 \mathrm{E}-4$.

Here the identification of $(P, S, P, P)$ and $(P, P, S, P)$ is very vague, but $\beta_{2}$, which is the proportion of $(P, S, S, P)$ to subtract from $\operatorname{tr}_{i_{2}}^{c}$, is almost its correct value of 1 , and the estimate of $v_{s, 2}$ is very good. Thus, for this noisy data where it is impossible to distinctly identify two of the ray types, one ray type is correctly identified, and the velocity estimates are good.

Conclusions. We have outlined a new efficient method for determining the seismic rays in a three-dimensional layered medium. This method can be included as the nucleus of an efficient nonlinear least squares algorithm for the estimation of medium parameters. Further details and applications of this method can be found in Fawcett [1].

\section{REFERENCES}

[1] J. FAWCETT, I. Three dimensional ray-tracing and ray-inversion in layered media. II. Inverse scattering and curved ray tomography with applications to seismology. Ph.D. thesis, California Institute of Technology, Pasadena, 1983.

[2] H. GJOYSTADTL AND B. URSIN, Inversion of reflection times in three dimensions, Geophysics, 46 (1981), pp. 972-983.

[3] H. B. Keller ANd D. J. Perozzi, Fast seismic ray tracing, this Journal, 43 (1983), pp. 981-992.

[4] C. T. LAwson And R. J. Hanson, Solving Least Squares Problems, Prentice-Hall, Englewood Cliffs, NJ, 1974.

[5] D. J. Perozzi, I. Seismic ray tracing in piecewise homogeneous media. II. Analysis of optimal step size selection in homotopy and continuation methods. Ph.D. thesis, California Institute of Technology, Pasadena, 1980.

[6] P. M. SHAH, Ray tracing in three dimensions, Geophysics, 38 (1973), pp. 600-604. 